\title{
Autonomous Lavatory Cleaning System
}

\author{
A. Adithya Bharadwaj, C. Arun Kumar, R. Balasubramanian, P. Gowtham \\ ECE Department, SSN College of Engineering, India
}

\begin{tabular}{l} 
Article Info \\
\hline Article history: \\
Received Jul 18, 2015 \\
Revised Oct 29, 2015 \\
Accepted Nov 19, 2015 \\
\hline Keyword: \\
Autonomous Lavatory \\
Cleaning System \\
RFID
\end{tabular}

\begin{abstract}
The application of integrated robotics is becoming increasingly commonplace in day to day applications. The idea presented in this paper seeks to provide a convenient and a hassle-free means of cleaning public toilets whilst maintaining hygienic and sanitary standards. By using a counter to record the number of times of usage, a line follower mechanism to guide the robot and an RFID module to initiate the flushing, the cleaning operation is fully automated and requires low operational power. Furthermore, a robotic arm is part of the module, so as to thoroughly clean the toilet bowl. Such a provision will curtail the role of manpower in maintenance of public toilets to a great degree, and thus, serves as a win-win situation; a revolting objective is accomplished with considerable ease.
\end{abstract}

Copyright $@ 2015$ Institute of Advanced Engineering and Science. All rights reserved.

Corresponding Author:

A. Adithya Bharadwaj,

ECE Department,

SSN College of Engineering,

Chennai, India.

Email: gowthamp151@gmail.com

\section{INTRODUCTION}

Urban sanitation is of prime importance in today's society and there exist a number of obstacles that need to be conquered. An easy to implement, yet effective method to facilitate the preservation of hygienic standards would be a welcome idea, and help overcome the various hurdles faced, such as human reluctance to engage in such a task. With this in mind, a viable idea would be to design a robot that is fully automated in functioning is competent in performance. Other considerations include ease of operation, power requirements and financial effectiveness.Using a low power microcontroller and a simple yet adequate line follower robot, this idea is very much practically realizable.

\section{RELATED WORKS}

The concept of using a robot for the purpose of sanitation can draw many parallels to present day technologies: robots are now used to serve a range of purposes, such as firefighting, dishwashing, search and rescue mission, to name some. At present, the cleaning process is entirely manual and not very productive. There exist very few methods similar to the one proposed.

\section{IMPLEMENTATION}

The robot is realized with the help of two microcontroller units (both MSP430).The first one (referred to asMCU-1) is present on the robot and helps in the movement and cleaning action of the robot. The second MCU (referred to asMCU-2) is interfaced with all the 4 cubicles and helps keep track of the counters. To initiate the cleaning mechanism once the robot reaches the desired cubicle, an RFID module is 
used. The robot is powered by a $12 \mathrm{~V}$ DC battery and each of the MCUs by independent $5 \mathrm{~V}$ sources. The working of this robot can be split into four modules as follows:

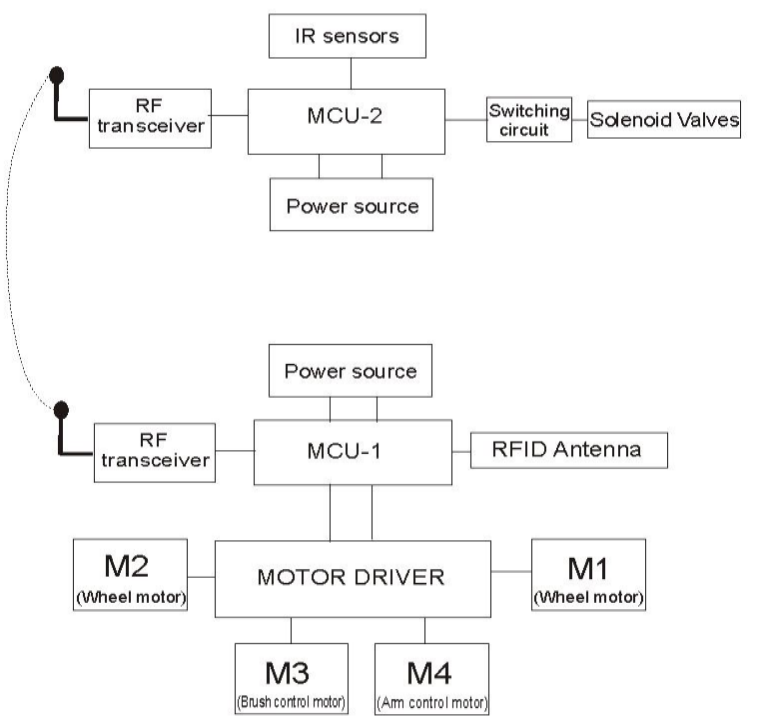

Figure 1. Block Diagram

\subsection{Module 1: Cubicle Selection}

Define The cubicle to be cleaned is selected based on the frequency of usage of that cubicle. The number of people visiting a particular cubicle is sensed by an Infrared transmitter-receiver pair which is placed at the doorway. Whenever a person enters a cubicle, he crosses this infrared sensor, causing it to become low. In all such instances, the counter corresponding to that cubicle is incremented. All such infrared sensors installed in each of the doorways is interfaced with an MSP430 MCU. This MCU-2 monitors the counter values and when one (or more) of the counters becomes greater than or equal to 5 , it sends the information regarding that cubicle to the robot (MCU-1) via an RF transmission module. When more than one counter value becomes greater than or equal to 5, then the cubicle with the largest count value is selected. If more than one counter has the largest value, then one of those cubicles is selected at random. After, the cubicle is selected, its counter value is reset to zero.

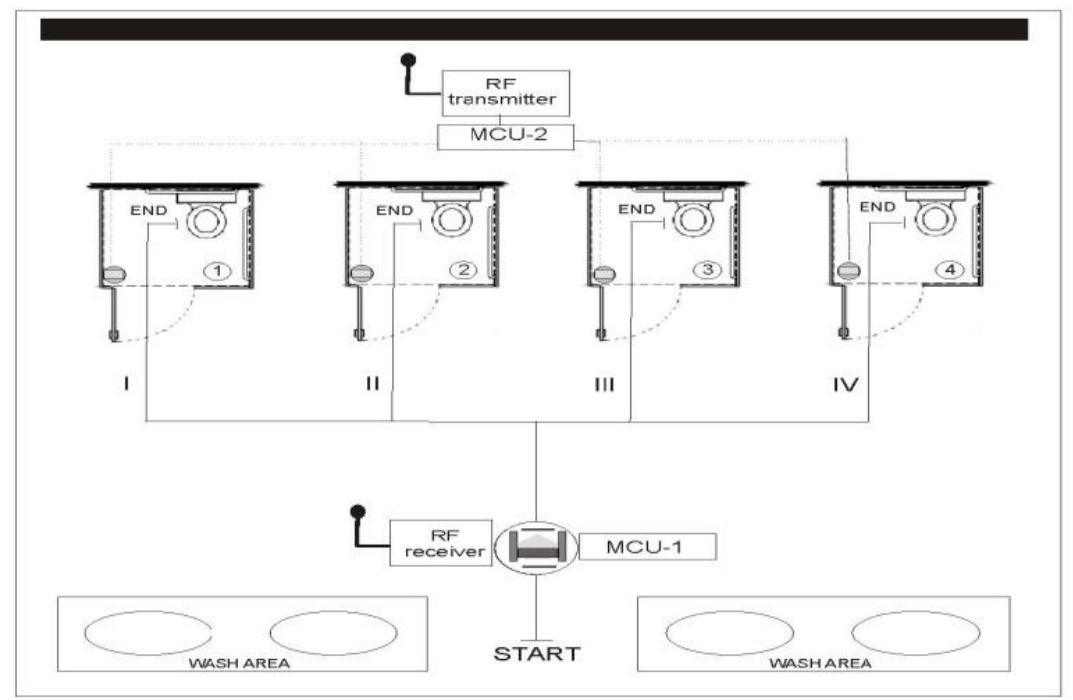




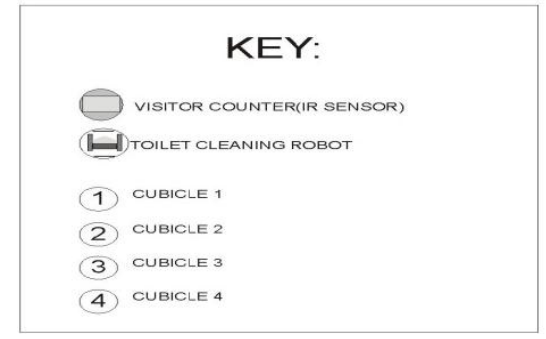

Figure 2. Locomotion Sequence

\subsection{Module 2: Locomotion}

The path from the starting point to each of the cubicles is laid as shown in Figure 1. Based on the data received from MCU-2,the cubicle and its corresponding path are chosen.The robot uses line follower technique to travel in the selected path. The robot has three wheels, two of which are connected to two 12-V DC motors(motor-1 and motor-2). The third wheel is a free wheel. These DC motor are interfaced with MCU1.By controlling the two motors, the robot can be made to move forwards,backwards,left or right. The path is a black line of thickness $5 \mathrm{~cm}$. The robot has 7 infrared sensors on its face. When a sensor faces the black line ,it sends a low output. Based on the data received from all the seven sensors, the robot is calibrated and prevents it from digressing. The robot sense the starting/ending point when all its seven sensors go low. Based on the path chosen, the robot makes a call on what turn to take at intersections. For example, if the path chosen is 'Path II', Then the robot takes a left turn on the first intersection and a right turn on the second. By this procedure, the robot reaches the desired cubicle.

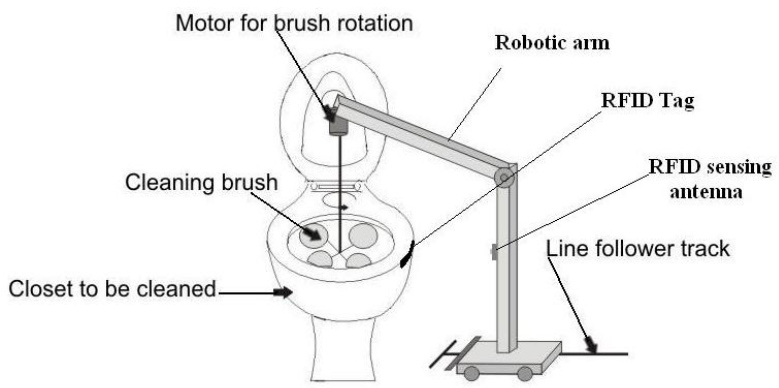

Figure 3. Cleaning Mechanism

\subsection{Module 3: Cleaning}

Once the robot reaches the destination it initiates the cleaning sequence. An RFID sensing antenna is affixed onto the robot; this senses the RFID tag placed on the toilet bowl, so as to trigger timers T1 and T2 simultaneously, associated with timers MCU-1 and MCU-2 respectively. Once the timers are triggered, the cleaning procedure is initiated, as explained below.

a. The toilet is automatically flushed with the help of a solenoid valve connected to the water supply (interfaced with MCU-2).

b. The arm containing the brush is lowered with the help of a 12-v DC motor (motor-3).

c. A cleaning liquid is dispensed into the walls of the closet and the axel containing the brushes is rotated using motor 4(12V-DC) to perform cleaning. This action happens for 35 seconds.

d. After 35 seconds, the brush is removed and the toilet is automatically flushed again.

e. The brush is again lowered in to the toilet and rotated for 25 seconds to perform thorough cleaning.

f. After 25 seconds, the brush is removed and the toilet flushed again.

Upon the completion of the above six steps, the timer runs out indicating the completion of the cleaning action. The counter for this particular cubicle is made zero.

\subsection{Module 4: Go Back and Repeat}

After the cleaning action is completed, the robot turns around and traces its path back to its initial position. The above process is repeated for as long as the robot is active. 

Figure 3.

The systematic working of the above modules can be pictorially represented using as shown in

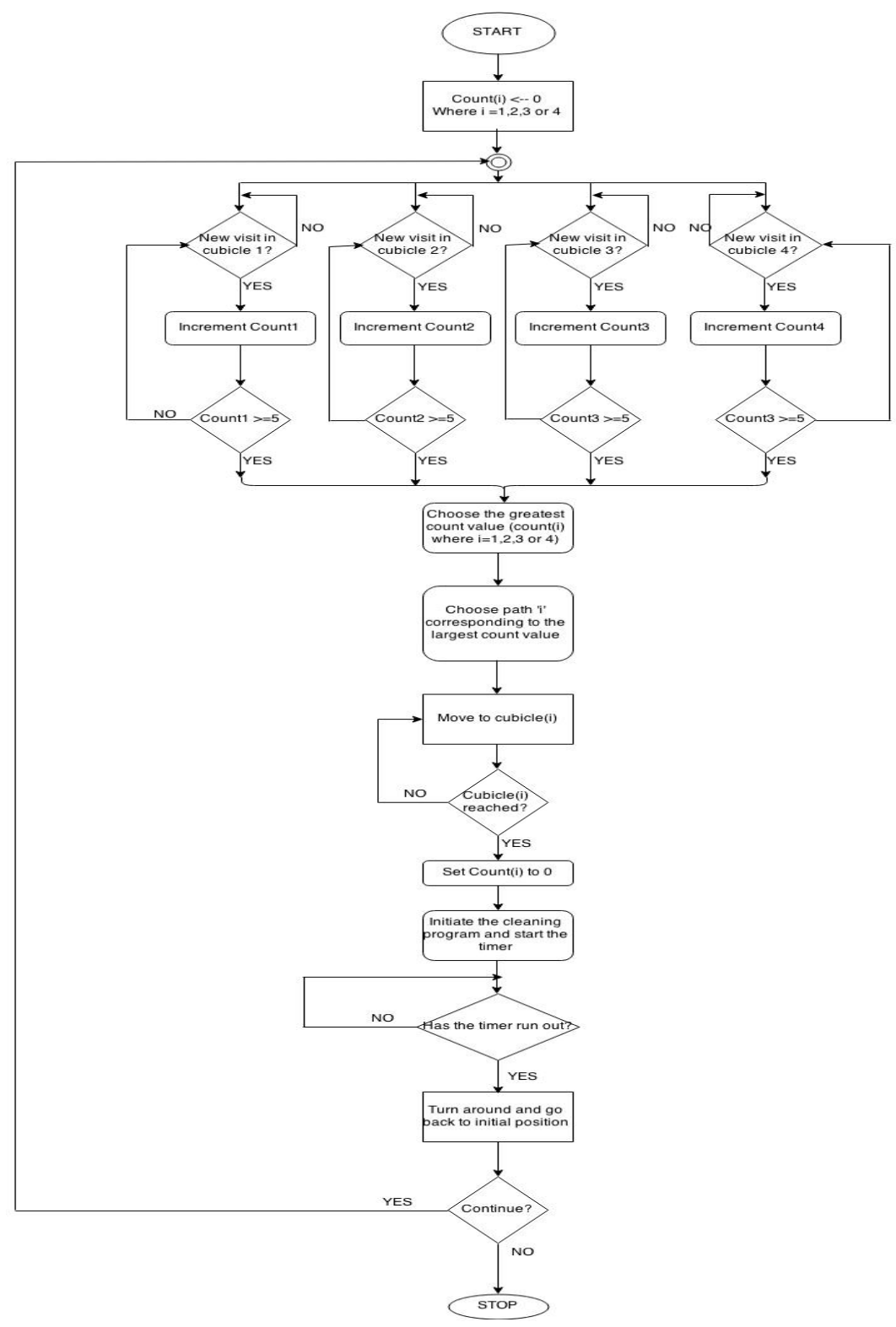

Figure 3. Systematic Working

\subsubsection{Future Advancements}

After the robot is implemented successfully, the robot can be improved by adding other cleaning modules to clean the other parts of the bathroom like the wash basins and walls. This added feature will make the robot a complete package and then the robot can be commercially sold as a wholesome package to substitute manual cleaning of restrooms.

\subsubsection{Advantages}

a. Low power and cost effective modules:

The MSP 430 used consumes low power and is highly cost effective compared to the higher end ATMEGA controllers used in Arduino platform.

b. Highly automated system:

The human element in the system is minimized to the level of the sporadic cleaning of the brushes and refilling of the cleaning liquid. 
c. Implementing RFID module is highly beneficial in a number of ways; it is cost effective and does not require a line-of-sight communication, possesses high speeds of operation and is best suited in tough environments.

\section{REFERENCES}

[1] Mohammed Iskatul Islam, Design and Fabrication Of A Line Follower Robot, 2011.

[2] John H. Davis, MSP 430 Microcontroller basics, Newnes, 2008.

[3] Jong-Hwan Kim, Eric T.Matson, Hyun Myung, Peter Xu, Fakhri Karray, Robot Intelligence Technology And Applications 2: Results from the 2nd International Conference On Robot Intelligence Technology And Applications, 2014 